\title{
Quartic diophantine chains
}

\author{
by
}

Ajai Choudhry (New Delhi) and JarosŁaw Wróblewski (Wrocław)

Dedicated to Professor A. Schinzel on his 70th birthday

Introduction. Very little is known about quartic diophantine chains of the type

$$
f\left(x_{1}, y_{1}\right)=f\left(x_{2}, y_{2}\right)=f\left(x_{3}, y_{3}\right),
$$

where $f(x, y)$ is a binary quartic form in the variables $x$ and $y$. For instance, only one numerical solution of the diophantine chain

$$
X_{1}^{4}-Y_{1}^{4}=X_{2}^{4}-Y_{2}^{4}=X_{3}^{4}-Y_{3}^{4}
$$

has been published [7, p. 652].

This paper is concerned with diophantine chains of the type

(3) $x_{1} y_{1}\left(x_{1}^{2}+r x_{1} y_{1}+y_{1}^{2}\right)=x_{2} y_{2}\left(x_{2}^{2}+r x_{2} y_{2}+y_{2}^{2}\right)=x_{3} y_{3}\left(x_{3}^{2}+r x_{3} y_{3}+y_{3}^{2}\right)$, where $r$ is an arbitrary rational number. The case $r=0$ is of special interest since then the chain (3) reduces to

$$
x_{1} y_{1}\left(x_{1}^{2}+y_{1}^{2}\right)=x_{2} y_{2}\left(x_{2}^{2}+y_{2}^{2}\right)=x_{3} y_{3}\left(x_{3}^{2}+y_{3}^{2}\right),
$$

which is equivalent to the chain of biquadrates

(5) $\left(x_{1}+y_{1}\right)^{4}-\left(x_{1}-y_{1}\right)^{4}=\left(x_{2}+y_{2}\right)^{4}-\left(x_{2}-y_{2}\right)^{4}=\left(x_{3}+y_{3}\right)^{4}-\left(x_{3}-y_{3}\right)^{4}$.

We will find a parametric solution of (3) for all non-zero values of $r$, and infinitely many integer solutions when $r=0$. In view of the equivalence of (4) and (5), the infinitely many solutions of (4) lead to infinitely many solutions of the chain of biquadrates (2).

We will regard a diophantine chain of type (3) as trivial if the common value $x_{i} y_{i}\left(x_{i}^{2}+r x_{i} y_{i}+y_{i}^{2}\right), i=1,2,3$, is 0 . Such chains are readily determined and will not be considered further in this paper. When the common value $x_{i} y_{i}\left(x_{i}^{2}+r x_{i} y_{i}+y_{i}^{2}\right)$ is not 0 , we have the following two types of chains:

2000 Mathematics Subject Classification: Primary 11D25. 
(i) if the absolute value of one of the numbers $x_{i}, y_{i}$ is the same positive number for each of the three values of $i$, and the absolute values of the three remaining numbers out of the six numbers $x_{i}, y_{i}, i=1,2,3$, are distinct, the chain will be called semi-trivial;

(ii) if the absolute values of all the six numbers $x_{i}, y_{i}, i=1,2,3$, are distinct, the chain will be called non-trivial.

In Section 2 below we obtain a necessary and sufficient condition for the existence of semi-trivial chains of type (3), and show how the complete solution may be obtained when this condition is satisfied. In Section 3 we obtain non-trivial diophantine chains for all rational values of $r$, and in Section 4 we give numerical solutions of the chain of biquadrates (2). As an application of this chain of biquadrates, in Section 5 we obtain infinitely many triads of biquadrates with equal sums and equal products.

We note that as equations (3) and (4) are both homogeneous, it suffices to obtain their solutions in rational numbers since any such solution may be multiplied through by a suitable integer to yield a solution in integers.

2. Semi-trivial quartic diophantine chains. In this section we will determine the complete solution of semi-trivial chains of type (3). Since equation (3) is of even degree, and in view of its symmetry, there is no loss of generality in assuming that for semi-trivial chains we may take $x_{1}=x_{2}=x_{3}$. With this assumption, (3) reduces to

$$
y_{1}\left(x_{1}^{2}+r x_{1} y_{1}+y_{1}^{2}\right)=y_{2}\left(x_{1}^{2}+r x_{1} y_{1}+y_{2}^{2}\right)=y_{3}\left(x_{1}^{2}+r x_{1} y_{3}+y_{3}^{2}\right) .
$$

The first part of equation (6) reduces to

$$
x_{1}^{2}+r x_{1}\left(y_{1}+y_{2}\right)+y_{1}^{2}+y_{1} y_{2}+y_{2}^{2}=0
$$

and we similarly get

$$
x_{1}^{2}+r x_{1}\left(y_{1}+y_{3}\right)+y_{1}^{2}+y_{1} y_{3}+y_{3}^{2}=0 .
$$

Now (7) may be rewritten as

$$
\left(4-r^{2}\right) X^{2}+Y^{2}+\left(3-r^{2}\right) Z^{2}=0
$$

where

$$
X=2 x_{1}+r\left(y_{1}+y_{2}\right), \quad Y=\left(4-r^{2}\right) y_{1}+\left(2-r^{2}\right) y_{2}, \quad Z=2 y_{2} .
$$

The solvability of the quadratic equation (9) is determined by the wellknown Legendre theorem [5, pp. 272-274], and when the condition of solvability is satisfied, the complete solution of (9), and hence of (7), can be effectively determined. We note in particular that when $r<\sqrt{3}$, the lefthand side of (9) is always positive for real values of $X, Y, Z$, not all 0 , and hence there are no non-trivial solutions of (9). 
Next we assume that equation (7) has non-trivial solutions. In this case the remaining condition for the existence of the chain (6), that is, equation (8), needs to be solved together with (7). Subtracting (7) from (8), and removing the non-zero factor $y_{2}-y_{3}$, we obtain a linear equation which is readily solved to get the unique solution $y_{3}=-r x_{1}-y_{1}-y_{2}$.

Thus for any given value of $r$, we can determine whether or not there exist semi-trivial solutions of (3) by determining the solvability of (9). When this condition is satisfied, all semi-trivial chains of type (3) are given by the complete solution of the quadratic equation (7) together with $x_{2}=x_{1}, x_{3}=$ $x_{1}, y_{3}=-r x_{1}-y_{1}-y_{2}$.

As a specific example, when $r=4$, we get the following parametric solution of (3):

$$
\begin{gathered}
x_{1}=x_{2}=x_{3}=p^{2}+3 p q+3 q^{2}, \\
y_{1}=-3 p^{2}-6 p q-2 q^{2}, \quad y_{2}=p^{2}+4 p q+q^{2}, \quad y_{3}=-2 p^{2}-10 p q-11 q^{2} .
\end{gathered}
$$

3. Non-trivial quartic diophantine chains. We first prove a preliminary lemma that will be used repeatedly to obtain solutions of the chain (3).

Lemma. If $a, b, c, d$ are non-zero rational numbers satisfying the simultaneous diophantine equations

$$
\begin{aligned}
& a\left(a^{2}+a b r+b^{2}\right)-c d=0, \\
& 3 a^{2}+2 a b r+b^{2}-c^{2}+d^{2}+1=0,
\end{aligned}
$$

then the rational numbers $x_{1}, y_{1}, x_{2}, y_{2}, x_{3}, y_{3}$ defined by

$$
x_{1}=b c, \quad y_{1}=a c-d, \quad x_{2}=b d, \quad y_{2}=a d+c, \quad x_{3}=b, \quad y_{3}=a+c d
$$

satisfy the diophantine chain (3).

Proof. When $x_{1}, y_{1}, x_{2}, y_{2}$ are defined by (12), we have

$$
\begin{aligned}
& x_{1} y_{1}\left(x_{1}^{2}+r x_{1} y_{1}+y_{1}^{2}\right)-x_{2} y_{2}\left(x_{2}^{2}+r x_{2} y_{2}+y_{2}^{2}\right) \\
& =b\left(c^{2}+d^{2}\right)\left[\left(c^{2}-d^{2}\right)\left\{a\left(a^{2}+a b r+b^{2}\right)-c d\right\}\right. \\
& \left.-c d\left(3 a^{2}+2 a b r+b^{2}-c^{2}+d^{2}+1\right)\right]=0,
\end{aligned}
$$

in view of (10) and (11). Similarly,

$$
\begin{aligned}
& x_{1} y_{1}\left(x_{1}^{2}+r x_{1} y_{1}+y_{1}^{2}\right)-x_{3} y_{3}\left(x_{3}^{2}+r x_{3} y_{3}+y_{3}^{2}\right) \\
& =b\left(c^{2}+1\right)\left[\left(c^{2}-1\right)\left\{a\left(a^{2}+a b r+b^{2}\right)-c d\right\}\right. \\
& \left.-c d\left(3 a^{2}+2 a b r+b^{2}-c^{2}+d^{2}+1\right)\right]=0 .
\end{aligned}
$$

Combining (13) and (14), we get the chain (3). This completes the proof. 
In view of the above lemma, to obtain rational solutions of the diophantine chain (3), it suffices to solve the simultaneous diophantine equations (10) and (11). This leads to two cases according as $r$ is non-zero or zero. We consider the case when $r \neq 0$ in Subsection 3.1 below and the case $r=0$ in Subsection 3.2.

3.1. To obtain rational solutions of equations (10) and (11) when $r \neq 0$, we solve (10) for $d$ to get

$$
d=a\left(a^{2}+a b r+b^{2}\right) / c
$$

and substituting this value of $d$ in (11), we get the following quartic equation in $c$ :

$$
c^{4}-\left(3 a^{2}+b^{2}+2 a b r+1\right) c^{2}-a^{2}\left(a^{2}+a b r+b^{2}\right)^{2}=0 .
$$

This may be considered as a quadratic equation in $c^{2}$, and for it to have a rational solution for $c^{2}$, its discriminant, namely,

$$
\begin{aligned}
\left(4 a^{2}+1\right) b^{4}+4 a r\left(2 a^{2}\right. & +1) b^{3}+\left\{4 a^{2} r^{2}\left(a^{2}+1\right)+8 a^{4}+6 a^{2}+2\right\} b^{2} \\
& +4 a r\left(2 a^{2}+1\right)\left(a^{2}+1\right) b+\left(4 a^{2}+1\right)\left(a^{2}+1\right)^{2},
\end{aligned}
$$

must be made a perfect square. By choosing $a=\left(t^{2}-1\right) /(4 t)$, the discriminant (17) becomes a quartic function of $b$ in which the constant term is a perfect square, and hence following the usual procedure described by Dickson [3, p. 639], we can obtain a value of $b$ which makes this quartic function a perfect square. With this value of $b$, equation (16) gives rational values for $c^{2}$ but not for $c$. If, however, we take $t=q^{2} / p^{2}$, then (16) has a rational solution for $c$. Thus, by taking

$$
a=-\left(p^{4}-q^{4}\right) /\left(4 p^{2} q^{2}\right),
$$

when we get the following value of $b$ which makes (17) a perfect square:

$$
\begin{aligned}
b= & \left\{\left(p^{4}-q^{4}\right)^{4} p^{4} q^{4} r^{2}+\left(p^{4}+q^{4}\right)^{2}\left(p^{8}+6 p^{4} q^{4}+q^{8}\right)^{2}\right\} \\
& \times\left\{4 p^{2} q^{2} r\left(p^{4}-q^{4}\right)\left(p^{4}+q^{4}\right)^{2}\left(p^{8}+6 p^{4} q^{4}+q^{8}\right)\right\}^{-1},
\end{aligned}
$$

we can solve (16) to obtain the following rational solution for $c$ :

$$
\begin{aligned}
c=\{ & \left\{\left(p^{8}-p^{6} q^{2}+4 p^{4} q^{4}-p^{2} q^{6}+q^{8}\right)\left(p^{2}-q^{2}\right)^{4}\left(p^{2}+q^{2}\right)^{2} p^{2} q^{2} r^{2}\right. \\
& \left.+\left(p^{4}+q^{4}\right)^{2}\left(p^{8}+6 p^{4} q^{4}+q^{8}\right)^{2}\right\} \\
& \times\left\{8 p^{3} q^{3} r\left(p^{2}-q^{2}\right)\left(p^{4}+q^{4}\right)^{2}\left(p^{8}+6 p^{4} q^{4}+q^{8}\right)\right\}^{-1} .
\end{aligned}
$$

Substituting the values of $a, b, c$ given by (18)-(20) in (15), we get

$$
\begin{aligned}
d=\{ & \left\{\left(p^{8}+p^{6} q^{2}+4 p^{4} q^{4}+p^{2} q^{6}+q^{8}\right)\left(p^{2}+q^{2}\right)^{4}\left(p^{2}-q^{2}\right)^{2} p^{2} q^{2} r^{2}\right. \\
\left.-\left(p^{4}+q^{4}\right)^{2}\left(p^{8}+6 p^{4} q^{4}+q^{8}\right)^{2}\right\} & \\
& \times\left\{8 p^{3} q^{3} r\left(p^{2}+q^{2}\right)\left(p^{4}+q^{4}\right)^{2}\left(p^{8}+6 p^{4} q^{4}+q^{8}\right)\right\}^{-1} .
\end{aligned}
$$


Thus a solution of (10) and (11) when $r$ is an arbitrary non-zero rational number is given in terms of arbitrary parameters $p$ and $q$ by (18)-(21). With these values of $a, b, c, d$, the values of $x_{i}, y_{i}, i=1,2,3$, defined by (12) constitute a parametric solution of the diophantine chain (3). It is readily verified that this parametric solution actually generates non-trivial diophantine chains of type (3) for every non-zero value of $r$.

3.2. We will now obtain infinitely many solutions of the diophantine chain (4). In view of the Lemma, it is sufficient to solve the following two simultaneous diophantine equations obtained by substituting $r=0$ in equations (10) and (11):

$$
\begin{aligned}
& a\left(a^{2}+b^{2}\right)-c d=0, \\
& 3 a^{2}+b^{2}-c^{2}+d^{2}+1=0 .
\end{aligned}
$$

We proceed as in Subsection 3.1 and instead of (16), we get the following equation obtained by substituting $r=0$ in (16):

$$
c^{4}-\left(3 a^{2}+b^{2}+1\right) c^{2}-a^{2}\left(a^{2}+b^{2}\right)^{2}=0 .
$$

As in Subsection 3.1, we must make (17) a perfect square when $r=0$, that is, we must solve the following quartic equation in $b$ and $s$ :

$$
s^{2}=\left(4 a^{2}+1\right) b^{4}+\left(8 a^{4}+6 a^{2}+2\right) b^{2}+\left(4 a^{2}+1\right)\left(a^{2}+1\right)^{2} .
$$

As before, we take

$$
a=\left(t^{2}-1\right) /(4 t)
$$

in $(25)$, and we get

$$
\begin{aligned}
s^{2}=\left\{256\left(t^{2}+1\right)^{2} t^{4} b^{4}+\right. & 32\left(t^{8}+8 t^{6}+46 t^{4}+8 t^{2}+1\right) t^{2} b^{2} \\
& \left.+\left(t^{2}+1\right)^{2}\left(t^{4}+14 t^{2}+1\right)^{2}\right\} /\left(1024 t^{6}\right) .
\end{aligned}
$$

The birational transformation defined by the relations

$$
\begin{aligned}
& b=Y /\left(4 t\left(t^{2}+1\right) X\right), \\
& s=\left\{-4 t^{2}\left(t^{2}-1\right)^{2}\left(t^{4}+6 t^{2}+1\right)^{2}-X^{2}\right\} /\left\{32 t^{3}\left(t^{2}+1\right) X\right\},
\end{aligned}
$$

and

$$
\begin{aligned}
X= & 8 t^{2}\left(t^{2}+1\right)^{2} b^{2}-16 t^{3}\left(t^{2}+1\right) s \\
& +\left(t^{8}+8 t^{6}+46 t^{4}+8 t^{2}+1\right) / 2, \\
Y= & 32 t^{3}\left(t^{2}+1\right)^{3} b^{3}-64 t^{4}\left(t^{2}+1\right)^{2} b s \\
& +2 t\left(t^{2}+1\right)\left(t^{8}+8 t^{6}+46 t^{4}+8 t^{2}+1\right) b,
\end{aligned}
$$


reduces the quartic equation (27) to the parametrized elliptic curve

$$
Y^{2}=X\left\{X+4 t^{2}\left(t^{2}-1\right)^{2}\right\}\left\{X-\left(t^{4}+6 t^{2}+1\right)^{2}\right\} .
$$

Now on using (27), (28) and (30), equation (24) gives

$$
\begin{aligned}
c^{2}= & \left(3 a^{2}+b^{2}+1 \pm s\right) / 2 \\
= & \mp(t \mp 1)^{2}\left\{X-4 t^{6}-24 t^{4}-4 t^{2} \mp\left(2 t^{7}+14 t^{5}+14 t^{3}+2 t\right)\right\}^{2} \\
& \times\left\{64 t^{3}\left(t^{2}+1\right)^{2} X\right\}^{-1} .
\end{aligned}
$$

For $c$ to be rational, we must find points on the curve (30) such that $\mp t X$ is a perfect square. It follows from the duplication formula [6, p. 31] that if there exists a rational point $P$ on the elliptic curve (30), then the abscissa of the point $2 P$ is a perfect square. It is therefore simplest to take $t=T^{2}$ in (30) and find a value of $T$ such that the curve (30) has a rational point. While $T=1$ leads to triviality, it was found using APECS (a package written by Connell [2] in MAPLE for working with elliptic curves) that when $T=2$ and also when $T=3$, there exist rational points on the elliptic curve (30). We will take $T=3$, that is, $t=9$, since this leads to numerically smaller solutions of the diophantine chains (2) and (4). With this value of $t$, the curve (30) becomes

$$
Y^{2}=X^{3}-47600704 X^{2}-103004636774400 X,
$$

and a rational point $P_{1}$ on this curve, found using APECS, is given by

$$
(X, Y)=(-3087587840 / 1521,126935576104960 / 59319) .
$$

As this rational point does not have integer co-ordinates, it follows from the Nagell-Lutz theorem [6, p. 56] on elliptic curves that this is not a point of finite order. Thus, there exist infinitely many rational points on the curve (32) and these can be determined by the group law. By using the duplication formula, we can find on this curve infinitely many rational points whose abscissae are perfect squares, and these rational points yield infinitely many rational solutions of equations (22) and (23) by using the relations (26), (28), (31) and (15) with $r=0$. We note that in all these solutions of (22) and (23), the value of $a$ remains fixed as 20/9. By the Lemma, the infinitely many solutions of (22) and (23) yield infinitely many rational solutions of the diophantine chain (4) and these, in turn, lead to infinitely many integer solutions of (4) as well as of (2).

We now prove that the above method actually generates infinitely many non-trivial chains of type (4). It is readily seen that the chains generated satisfy $x_{i} y_{i}\left(x_{i}^{2}+y_{i}^{2}\right) \neq 0, i=1,2,3$. The only remaining possibility for a chain to be trivial arises when we have $x_{i} y_{i}=x_{j} y_{j}$ for some $i \neq j$. In each of these cases the numbers $a, b, c, d$ must satisfy another equation in addition to the equations (22) and (23). Eliminating $c$ and $d$ from the three equations in $a, b, c, d$, we get an equation in $a$ and $b$ which for a fixed non-zero 
$a$ has only finitely many solutions in $b$. Since there are only three such sets of three equations in $a, b, c, d$, we can simply exclude the resulting finitely many possibilities from the infinite set of solutions with $a=20 / 9$, and thus obtain infinitely many non-trivial diophantine chains of type (4). The possibility that these infinitely many non-trivial diophantine chains are actually multiples of a finite number of such chains is also similarly ruled out. We are thus assured of generating infinitely many non-trivial solutions of (4), and hence also of (2).

While the above procedure guarantees the existence of infinitely many integer solutions of (2) and (4), the solutions generated involve large integers. For instance, using the rational point $P_{1}$ already found on the curve (32), we determine the point $2 P_{1}$ which is given by

$(X, Y)=\left(\frac{6867760262600^{2}}{274373463^{2}}, \frac{311333393877294196698891729982967296000}{20655053024783974477163847}\right)$.

As expected, the abscissa of $2 P_{1}$ is a perfect square, and this yields the following solution of the chain of biquadrates (2):

$$
\begin{aligned}
X_{1} & =481414016786139336958126813632656903003004 \\
X_{2} & =452693557121427726005012503163912114942985 \\
X_{3} & =591920656152766167797077466343528334914140 \\
Y_{1} & =336113147372142763632798008407011624578004 \\
Y_{2} & =179887595380427256472663477672099005579255 \\
Y_{3} & =534811611453397202401641358319895724884620 .
\end{aligned}
$$

Solutions that are much smaller than the above do exist, and can be obtained by numerical methods. These methods are discussed in the next section.

We have already noted that when $t=4$ and $t=9$, rational points on the elliptic curve (30) lead to solutions of the diophantine chain (2). There are several other elliptic curves, arising from equations (22) and (23), that similarly lead to integer solutions of (2). The existence of several such elliptic curves, together with the frequency of numerical solutions obtained in the next section, suggests the possibility of a parametric solution of (2). This, however, remains an open problem.

4. Chains of biquadrates. To obtain small solutions of (2) we search for small solutions of the equation

$$
A^{4}+B^{4}=C^{4}+D^{4} .
$$

One method to find small solutions of (35) is an exhaustive search. The key observation to speed it up is to consider two cases. 
(i) If there is one odd and one even term on each side of (35) then we can assume $A, C$ to be odd and $B, D$ to be even. It can be shown that in this case $A \equiv \pm C(\bmod 64)$ and $B \equiv D(\bmod 4)$.

(ii) If there are two odd terms on each side of (35) then we can assume $A^{4} \equiv C^{4}(\bmod 13)$ and $B^{4} \equiv D^{4}(\bmod 13)$.

In both cases pairs $(A, C)$ are stored in memory and checked against pairs $(B, D)$. We fix a prime $p$ and for each $0 \leq R<p$ we store at once only the pairs $(A, C)$ with $A^{4}-C^{4} \equiv R(\bmod p)$. We will not discuss the further details of the particular implementation used.

A search using a $\mathrm{C}$ program determined that there are 1420 primitive solutions of (35) with $A, B, C, D<10^{7}$. Case (i) took under 19 days on Pentium $41.7 \mathrm{GHz}$ and case (ii) took about 16 days with a 2-threaded program on Pentium 4 HT $2.4 \mathrm{GHz}$.

An easy check up of the results determined that the chain (2) has four primitive solutions with positive integers $X_{1}, Y_{1}, X_{2}, Y_{2}, X_{3}, Y_{3}<10^{7}$, and in the same range there is no chain

$$
X_{1}^{4}+Y_{1}^{4}=X_{2}^{4}+Y_{2}^{4}=X_{3}^{4}+Y_{3}^{4} .
$$

Another algorithm used to produce solutions of (35) is the method of Pythagorean triplets described in [7]. An exhaustive search for solutions to [7, eq. (10), p. 638] in the range $x, y, z \leq 40000$ resulted in 1592 solutions of (35), and a selective search in a higher range produced many more.

Every solution of (35) leads to numbers $x_{1}, y_{1}, x_{2}, y_{2}$ satisfying the left equation of (4) and we can use (12) to get complex numbers $x_{3}, y_{3}$ satisfying (4). If $x_{3}, y_{3}$ happen to be rational we get a rational chain (4) which leads to a chain (2).

This method produced 40 primitive integer chains (2), the largest having 14-digit terms.

We give 23 chains with terms below $10^{10}$ in Table I.

It was verified that among primitive solutions of (35) with $A, B, C, D<$ $10^{7}$ there are no two solutions with the same $\max (A, B, C, D)$.

Using the 14th chain in Table I we get two primitive solutions of (35) with $\max (A, B, C, D)=657153271$.

5. An application of the chains of biquadrates. Using chains (2) we can obtain an infinite family of solutions to the diophantine system

$$
\begin{aligned}
A^{4} B^{4} C^{4} & =D^{4} E^{4} F^{4}, \\
A^{4}+B^{4}+C^{4} & =D^{4}+E^{4}+F^{4},
\end{aligned}
$$

mentioned in [4, p. 142] and discussed in [1]. 
Table I. Solutions of the chain $X_{1}^{4}-Y_{1}^{4}=X_{2}^{4}-Y_{2}^{4}=X_{3}^{4}-Y_{3}^{4}$

\begin{tabular}{rrrrrr}
\hline$X_{1}$ & \multicolumn{1}{c}{$Y_{1}$} & \multicolumn{1}{c}{$X_{2}$} & \multicolumn{1}{c}{$Y_{2}$} & \multicolumn{1}{c}{$X_{3}$} & \multicolumn{1}{c}{$Y_{3}$} \\
\hline 335084 & 296668 & 265076 & 93436 & 264047 & 1169 \\
421296 & 273588 & 415137 & 248289 & 401168 & 17228 \\
854688 & 813396 & 747633 & 682161 & 614656 & 465236 \\
3138156 & 2840232 & 2377876 & 500296 & 2376783 & 249999 \\
27220940 & 24543080 & 20830065 & 6730545 & 20773068 & 39432 \\
43134160 & 39597700 & 37607745 & 31601025 & 32721072 & 19453572 \\
49734032 & 47450804 & 32004351 & 5106879 & 31999248 & 1829556 \\
49888344 & 39566652 & 44400113 & 19439153 & 43986552 & 1772484 \\
57218008 & 56255396 & 28999496 & 7923364 & 28962047 & 4144961 \\
80325288 & 41563476 & 79714568 & 36280748 & 79087329 & 26275617 \\
91785840 & 89603460 & 50547505 & 11071345 & 50518608 & 3231108 \\
140326844 & 139033552 & 61413863 & 18878503 & 61306948 & 12960784 \\
197237095 & 150215015 & 191610280 & 136159640 & 178105688 & 37694312 \\
657153271 & 613063351 & 469658376 & 241846092 & 461665368 & 117778356 \\
751888607 & 509011231 & 728659604 & 414173824 & 708852076 & 31047392 \\
1180872001 & 1161828737 & 616132528 & 383720564 & 601308944 & 301870196 \\
1596600137 & 1582784777 & 716243652 & 450436344 & 702011844 & 380055768 \\
3071712177 & 2980091889 & 1860358392 & 1161850644 & 1805476184 & 828030484 \\
4275254036 & 4274662012 & 660603913 & 271493129 & 660079796 & 263615324 \\
4779990264 & 4779753552 & 628280072 & 478478672 & 625923087 & 473081841 \\
6427460484 & 5799560808 & 5594408068 & 4483641784 & 4989936609 & 2584078431 \\
6621888824 & 6399150812 & 4017771432 & 1956085212 & 3968658927 & 1208210001 \\
9865447832 & 8250756572 & 8782377144 & 5772976932 & 8348157633 & 2076294399 \\
\hline & & & & &
\end{tabular}

If we take

$$
\begin{array}{ll}
A=X_{1} Y_{2}, & B=X_{2} Y_{3}, \quad C=X_{3} Y_{1}, \\
D=X_{1} Y_{3}, & E=X_{2} Y_{1}, \quad F=X_{3} Y_{2},
\end{array}
$$

then (37) is obvious, while (38) can be written as

$$
\operatorname{det}\left(\begin{array}{ccc}
X_{1}^{4} & X_{2}^{4} & X_{3}^{4} \\
Y_{1}^{4} & Y_{2}^{4} & Y_{3}^{4} \\
1 & 1 & 1
\end{array}\right)=0
$$

which is true since by (2) the difference of the first two rows is a multiple of the third one.

Acknowledgments. We are grateful to the referee for his comments which have led to improvements in the paper. 


\section{References}

[1] A. Choudhry, Triads of biquadrates with equal sums and equal products, Mathematics Student 70 (2001), 149-152.

[2] I. Connell, APECS, Arithmetic of Plane Elliptic Curves, http://www.math.mcgill.ca /connell/public/apecs/.

[3] L. E. Dickson, History of the Theory of Numbers, Vol. II, Chelsea, 1992, reprint.

[4] R. K. Guy, Unsolved Problems in Number Theory, Springer, New York, 1994.

[5] K. Ireland and M. Rosen, A Classical Introduction to Modern Number Theory, 2nd ed., Springer, New York, 1990.

[6] J. H. Silverman and J. Tate, Rational Points on Elliptic Curves, Springer, New York, 1992.

[7] A. J. Zajta, Solutions of the diophantine equation $A^{4}+B^{4}=C^{4}+D^{4}$, Math. Comp. 41 (1983), 635-659.

D-6/1, Multi-Storey Flats, Sector 13, R.K. Puram

Mathematical Institute

New Delhi 110066, India

E-mail: ajaic203@yahoo.com

University of Wrocław

Pl. Grunwaldzki 2/4

50-384 Wrocław, Poland

E-mail: jwr@math.uni.wroc.pl

Received on 16.10.2006

and in revised form on 22.4.2007 